\section{Carta al Editor}

\section{Sr. Editor Revista Chilena de Anestesia:}

Estimado Colega:

Se me solicitó escribir un artículo de revisión sobre metaanálisis para el número de bioestadística de vuestra revista. Al leer el artículo publicado noté que se hicieron correcciones sin notificarme, con lo cual aparecen bajo mi nombre, conceptos que a mi entender son erróneos y con los que no estoy de acuerdo. Considero impropio efectuar modificaciones de un manuscrito sin el

\section{Respuesta a Carta al Editor}

La carta del Dr. Dagoberto Ojeda D. manifiesta su disconformidad porque en un artículo de su autoría se incluyeron, sin su autorización, conceptos que él no compartía.

Efectivamente esto es así, y nuestra primera acción fue conversar personalmente con el Dr. Ojeda y tratar de explicar la razón por qué había ocurrido tal cosa, especialmente en un artículo que había sido escrito por encargo, pero el Dr. Ojeda no quiso aceptar estas explicaciones, por lo que corresponde, con todo derecho, dárselas por escrito. Además de las explicaciones al Dr. Ojeda, es una oportunidad para compartir con los lectores las dificultades y presiones a las que se ve sometido quien o quienes tienen la responsabilidad de publicar oportunamente los números de la revista.

La idea de hacer un número de la Revista Chilena de Anestesia dedicada a comprender el lenguaje estadístico y dar nociones de bioestadística y epidemiología para una lectura crítica de la literatura nació hace varios años con el Dr. Jorge Dagnino S. pues el tema, aunque había sido tratado, necesitaba una puesta al día. Diseñamos un temario y decidimos que era bueno que además del Dr. Dagnino como editor invitado, debíamos integrar a un par de anestesiólogos de la nueva ge- consentimiento del autor; si el contenido de un artículo es equivocado a juicio de los editores, este debe ser rechazado simplemente o correspondería advertir al autor que existen errores que él mismo debe corregir.

Por estas razones rogaría publicar esta carta, para que no se me achaquen conceptos que no comparto.

Atte.

Dagoberto Ojeda D. Anestesiólogo Clínica Dávila Magister Bioestadística Universidad de Chile

neración, que se integraran como coeditores. Precisamente el Dr. Dagoberto Ojeda, anestesiólogo de Clínica Dávila, por tratarse de una persona con un postgrado en Estadística en la Universidad de Chile y el Dr. David Torres Pérez, anestesiólogo de Clínica Santa María con un postgrado en Epidemiología en la Universidad de la Frontera. A ambos se les hizo consultas sobre el contenido, sobre posibles colaboradores en la autoría de los diferentes temas y se les encomendó, a elección, algunos temas a ellos mismos.

A pesar de que muchos artículos nunca llegaron y que el $80 \%$ del contenido de la futura Revista fue de autoría del Dr. Dagnino, el material que tuvimos alcanzó para dos números del volumen 43 de la Revista: 2 y 4 . Unos cuantos artículos no pudieron publicarse por la discreta calidad de su contenido y porque su reparación habría tomado demasiado tiempo.

La rutina habitual de publicación es recibir un artículo, enviarlo a evaluación por un miembro del consejo editorial y generalmente devolverlo al autor sugiriéndole algunas modificaciones para un mejor resultado final; algunas veces las modificaciones son de tal magnitud, que el trabajo se rechaza y las menos de las veces el trabajo se aprueba en forma inmediata. Siempre los artículos son envidiados en formato pdf para una revisión final del autor.

En los artículos hechos por encargo, como es 
el caso del artículo “¿Qué es un metaanálisis?” de los autores Dr. Dagoberto Ojeda y Dra. Javiera Wurth, los artículos generalmente son modificados a discreción por el editor, el coeditor y en este caso, además por el editor invitado. Talvez con la única excepción del artículo "Consentimiento informado en investigación" del Dr. Gonzalo Mutizábal (en que utiliza un lenguaje impecable), la totalidad de ellos fueron modificados de acuerdo a los criterios de los editores: desde la ortografía, la sintaxis, la redacción y hasta el contenido. Más de algún artículo incluso cambió su nombre después de la revisión.

En los dos números dedicados al tema, hicimos la innovación, al estilo British Journal y otras revistas, de resaltar al inicio los puntos considerados más destacables del capítulo. Les solicitamos a los autores enviar estos puntos pero en la mayoría de los casos nunca nos llegaron y tuvimos que hacerlo los editores. Además, como siempre, borramos algunas cosas y agregamos frases y conceptos menores aquí y allá.

El artículo de los Dres. Ojeda y Wurth sufrió algunos cambios que los editores creímos enriquecían el texto pero que fueron objetados por los autores. Quisiéramos dar una explicación, que dividiremos en forma y fondo:

1. En la forma, los autores tienen razón porque no recibieron el archivo en formato pdf final donde pudieron haber manifestado su desacuerdo y el artículo no se hubiera publicado, o lo más probablemente hubiéramos llegado a algún acuerdo porque un tema tan importante y de actualidad no hubiera podido dejarse afuera. El problema es que el número tenía que irse a prensa para salir con fecha diciembre y todas estas comunicaciones vía e-mail, con textos adjuntos van demorando enormemente el tiempo. Por esto no enviamos el texto definitivo a ningún autor, decisión que tomó el editor titular, por lo que pido públicamente disculpas a los autores de este artículo y otros sobre los cuales intervenimos sus textos, que hubieran podido molestarse, pero no lo manifestaron francamente como lo hace en su carta el Dr. Ojeda.

2. En el fondo, los autores se molestan por un párrafo específico y vamos a referirnos exclusivamente a esto y no a otra serie de modificaciones y correcciones de errores que no hubieran podido publicarse como estaban en el original y que no fueron objetads por los autores. Lo más objetivo sería que publicáramos el artículo como fue recibido junto al artículo editado y los lectores hicieran su propio juicio, pero como esto no es posible ni conveniente, nos centraremos en el quid del asunto.

Originalmente el texto decía:

"La medicina basada en la evidencia surgió como una manera de mantener el paso con el desarrollo acelerado de la ciencia médica y como un cambio del paradigma tradicional que reconocía en la intuición, la experiencia y el razonamiento fisiopatológico a árbitros idóneos para la toma de decisiones clínicas. Así se llegó a configurar una jerarquía de la evidencia científica que va desde los metaanálisis de ensayos clínicos en la cúspide hasta descender a las meras opiniones/observaciones clínicas". Se inserta allí una figura (no citada en el texto) en que aparece un triángulo con la revisión sistemática de ensayos clínicos con o sin metaanálisis en el vértice (señalado como el no plus ultra de la evidencia científica), seguido por los ensayos clínicos ( $\sin$ ningún apelativo) y terminando la pirámide con la opinión de expertos.

La verdad es que al leer el artículo completo daba la impresión que si un metaanálisis llegaba a una conclusión, no había forma de rebatir su resultado. En estos tiempos en que se publican metaanálisis de muy mala calidad, muchas veces basados en estudios no aleatorizados y/o con una escuálida muestra, el editor invitado sugirió que había que matizar la afirmación tan drástica del valor intocable de un metaanálisis en la actualidad. Era importante hacer una discusión de las limitaciones y problemas de los metaanálisis y realizar una lista de verificación o de consejos para que ayude a los lectores a distinguir un buen de un mal metaanálisis ${ }^{2,3}$.

Al estar casi en el límite del tiempo de entrega de la Revista y sabiendo que hay muchos quienes disienten de que el metaanálisis sea la cúspide de la evidencia científica, separando las investigaciones en prospectivas y retrospectivas (sólo entre estas, las revisiones sistemáticas ocuparían la cúspide), decidimos agregar una frase al texto, que es lo que constituye la mayor molestia del Dr. Ojeda. Es mucha la literatura que al discutir los metaanálisis, junto con resaltar lo 
que aportan y que los editores apoyan, discuten sus limitaciones; para muchos investigadores de fuste, un metaanálisis nunca es más fuerte que el más débil de los trabajos prospectivos que analiza. A modo de ejemplo, un estudio identificó metaanálisis cuyas conclusiones fueron desvirtuadas posteriormente por un estudio prospectivo de suficiente tamaño ${ }^{4}$. Para los editores era importante presentar a los lectores las dos visiones y no sólo la de los autores del artículo para quienes las revisiones sitemáticas y los metaanálisis son, sin discusión alguna, la cúspide de la evidencia científica. A nuestro parecer, presentar en el artículo sólo una parte de las opiniones y no la argumentación opuesta es precisamente el sesgo que las revisiones sistemáticas pretenden disminuir.

Es así como, manteniendo la figura exactamente igual, porque esa es efectivamente la opinión de Manchikanti y muchos otros autores, agregamos: "Sin embargo, no hay acuerdo unánime al respecto, $\mathrm{y}$ hay quienes prefieren no tomar las conclusiones de una revisión sistemática o un metaanálisis como verdades absolutas, o considerarlos casi como un sinónimo de evidencia. Parece más ecléctico separar las investigaciones en prospectivas y retrospectivas y considerar en la cúspide de la evidencia sólo las prospectivas. Para prevenir conclusiones equívocas de un metaanálisis es conveniente saber la forma de interpretarlos adecuadamente y conocer los posibles sesgos que pudieran invalidarlos, pues son de una variada calidad. Un buen ensayo aleatorizado y con un tamaño muestral adecuado, pudiera aportar mejor información y ser más beneficioso para cambiar una conducta clínica que un metaanálisis sesgado. Por otra parte, un buen metaanálisis de estudios metodológicamente mal diseñados dará lugar a malos resultados estadísticos".

Como el Dr. Ojeda era coeditor invitado y lo considerábamos formando parte de un equipo que trabajaba en conjunto para sacar adelante esta edición, consideramos innecesario, dado el apuro de entrega del material, confirmar su acuerdo con el texto agregado. Sentimos su molestia y volvemos a pedir disculpas, de igual forma que al Dr. Torres, a quien cambiamos, borramos y agregamos bastantes párrafos de aquéllos artículos a su cargo.

Tenemos un texto firmado por el propio Dr.
Ojeda que asevera textualmente, refiriéndose a 3 ensayos clínicos publicados en nuestra Revista y que fueron criticados negativamente en un editorial: "Los trabajos en cuestión eran ensayos clínicos, que constituyen el top de la evidencia de manera que habría que explicar que era lo erróneo en ellos que explicara el resultado erróneo; existe un checklist para verificar si un ensayo clínico está bien diseñado (CONSORT Statement) ${ }^{5}$ y la 'etiqueta' de la medicina basada en la evidencia nos exige basar las críticas al diseño de un ensayo clínico en esta guía"6. Con este antecedente, pensamos que lo agregado no va en contra de las convicciones del Dr. Ojeda.

Necesitábamos también darle consistencia al número completo. En el artículo sobre Revisiones Sistemáticas, en la misma Revista, la Dra. Rojas y el Dr. Torres habían manifestado: “...no hay que desmerecer los estudios controlados aleatorizados prospectivos, que bien ejecutados y con un buen número de pacientes pueden cambiar conductas clínicas. En cambio, una revisión sistemática mal hecha puede ser equívoca en sus conclusiones. Asimismo, si una revisión sistemática está mal hecha, un metaanálisis hecho a partir de ella también lo estará"7. Esto concuerda perfectamente con la opinión de los editores y es lo único que tratamos de manifestar: el metaanálisis no es una panacea.

Como anécdota, contaremos que los más difícil de este artículo fue ponernos de acuerdo en la terminología a usar, pues había tres maneras de escribir metaanálisis: meta-análisis, metanálisis o metaanálisis, como en definitiva se hizo. Al respecto, incluimos también un párrafo que no fue consultado con los autores del artículo. El Diccionario de la Real Academia Española, en una revisión somera, no ayuda. Como la tendencia actual es a no separar con guion los prefijos, el término meta-análisis quedó descartado. Sin embargo, hubo tres argumentos que llevaron al editor (con oposición del editor invitado quien insiste en la cacofonía) a terminar usando metaanálisis, y hacer consistente esa palabra en todo el número de la Revista, aunque visual y auditivamente nos gustara la palabra metanálisis.

Lo primero fue un sitio de Internet que hacía referencia a la tendencia actual a abandonar los guiones y mantener las dos vocales cuando se usan prefijos terminados en vocal (intraarti- 
cular, metaanfetamina). Además, aunque no nos gusta argumentar en una revista científica con Wikipedia, hay que reconocer que a veces sir$\mathrm{ve}^{8}$. Lo segundo fue que, aunque la literatura española estaba bastante dividida en los términos, en la literatura chilena encontramos un artículo de la Dra. Luz María Letelier, de la Unidad de Medicina Basada en la Evidencia de la PUC, en que se usaba el término metaanálisis 9 . Lo tercero, y quizás más sólido, es que según la nueva Ortografía de la Lengua Española ${ }^{10}$, los prefijos se escriben unidos a la base léxica, sin espacio y $\sin$ guion intermedio, cuando esta consta de una sola palabra. Ejemplos: expresidente; ultraactividad; exmujer; antivirus; panárabe; codirector; seudovacaciones; posguerra; poselectoral; postsocialismo (se prefiere pos a post, excepto cuando la palabra a la que se une el prefijo comienza por s); preelectoral, metaanálisis, psicoorientador (salvo excepciones, en la unión de un prefijo con una palabra no se simplifican las vocales); subbético, subboreal (en este caso sí se conservan las dos b, con la excepcuón de subranquial y subrigadier).
En resumen, en la forma le pedimos disculpas al Dr. Ojeda; la prisa por cumplir con los plazos y publicar a tiempo los números comprometidos, cuyo fin es indizar en algún momento nuestra Revista, y las demoras idiosincráticas que atentan contra ello y que constituyen una pesadilla para los editores. En cuanto al fondo, creemos tener la razón; si bien respetamos que el Dr. Ojeda considere erróneos los conceptos agregados en el cuestionado párrafo, tenemos la convicción que el párrafo agregado entrega a los lectores una visión balanceada y basada en la mejor evidencia disponible y cuyo respaldo en la literatura es mucho más amplio que las referencias que agregamos al artículo del Dr. Ojeda, y que aquí reproducimos agregando otras ${ }^{11-16}$. Esta es una misión irrenunciable de los editores.

\section{Dr. Ricardo Bustamante Bozzo \\ Editor}

Dr. Jorge Dagnino Sepúlveda

Editor Invitado

\section{Referencias}

1. Manchikanti L, Benyamin RM, Helm S, et al. Evidence-based medicine, systematic reviews, and guidelines in interventional pain management: part 3: systematic reviews and meta-analyses of randomized trials. Pain Physician 2009 JanFeb;12(1):35-72.

2. Greco T, Zangrillo, BiondiZoccai G, et al. Meta-analysis: pitfalls and hints. Heart Lung Vessel 2013;5:21-225.

3. Reade MC, Delaney A, Bailey $\mathrm{MJ}$, et al. Bench-to-bedside review: Avoiding pitfalls in critical care meta-analysis - funnel plots, risk estimates, types of heterogeneity, baseline risk and the ecologic fallacy.

4. LeLorier J, Grégoire G, Benhaddad A, et al. Discrepancies between meta-analyses and subsequent large randomized, controlled trials. N Engl J Med 1997 Aug;337(8):536-542.

5. Schulz KF, Altman DG, Moher D; CONSORT Group. CONSORT 2010 statement: updated guidelines for reporting parallel group randomised trials. BMJ 2010;340 mar23 1:c332.

6. Comunicación personal recibida por correo electrónico por el editor.

7. Rojas C, Torres D. Revisiones Sistemáticas. Rev Chil Anest; 2014. p. 42.

8. Wikipedia. Metaanálisis (Accesado en abril de 2015 en: https://es.wikipedia.org/wiki/ Metaan\%C3\%A1lisis).

9. Letelier LM, Manríquez JJ, Rada G. Revisiones sistemáticas y metaanálisis: ¿Son la mejor evidencia? Boletín de la Escuela de Medicina 2005;30:37-39.

10. Ortografía de la Lengua Española. Real Academia Española y Asociación de Academias de la Lengua Española: Espasa, 2010.

11. Moher D, Pham B, Jones A, et al. Does quality of reports of randomised trials affect estimates of intervention efficacy reported in meta-analyses? Lancet 1998 Aug;352(9128):609-613.

12. Streiner DL. I have the answer, now what's the question?: why metaanalyses do not provide definitive solutions. Can J Psychiatry 2005 Nov;50(13):829-831.

13. Garg AX, Hackam D, Tonelli M; When One Study Is Just Not Enough. Systematic review and meta-analysis: when one study is just not enough. Clin J Am Soc Nephrol 2008 Jan;3(1):253- 
260.

14. Walker E, Hernández AV, Kattan MW. Meta-analysis: its strengths and limitations. Cleve Clin J Med 2008 Jun;75(6):431-439.

15. Flather MD, Farkouh ME, Pogue
JM, et al. Strengths and limitations of meta-analysis: larger studies may be more reliable. Control Clin Trials 1997.

16. Bartolucci AA, Hillegass WB. Overview, Strenghts and Limita- tions of Systematic Reviews and Meta-Analysis. En Chiapelli F et al (eds). Evidence-Based Practice: Toward Optimizing Clinical Outcomes. Springer-Verlag: Berlin, 2010. Pp. 17-33. 